\title{
Líneas de acción para el diseño de herramientas de política pública contra la creciente demanda de energía para el enfriamiento de espacios en edificios
}

\section{Guidelines for public policies development against the growing demand of energy for space cooling in buildings}

ARTÍCULO

\author{
Hernán Baulo Darhanpé \\ Universidad Nacional de Mar del Plata, Argentina. Contacto: hernanbaulo@gmail.com
}

Recibido: agosto de 2020

Aceptado: octubre de 2020

\section{Resumen}

El presente artículo aborda la problemática derivada del incremento en la demanda de energía para enfriamiento de espacios en edificios, tendencia que se verifica a escala mundial. La ciudad de Buenos Aires y sus alrededores densamente poblados (AMBA) no escapan a los impactos negativos de esta tendencia, que se desplaza dentro de un círculo vicioso de demanda energética de una matriz mayormente dependiente de fuentes fósiles, por un lado, y eventos cada vez más frecuentes de calor extremo, por el otro. Ante esta problemática, que se da en el marco de complejos cambios en las pautas de consumo y producción a nivel global, este artículo propone lineamientos de acción para abordar de un modo sistémico los impactos ambientales derivados del creciente uso de energía en edificios: posibles intervenciones sobre el mercado de sistemas activos para acondicionamiento térmico; metodologías que permitan actuar en términos normativos sobre aspectos pasivos de las edificaciones; y la transición hacia una mayor incorporación de fuentes energéticas renovables.

Palabras claves: ambiente, cambio climático, energía, desarrollo sustentable, innovación, arquitectura bioclimática.

\footnotetext{
Abstract

This article addresses the problems derived from the increase in energy demand for space cooling in buildings - a trend that is verified globaly -, the recent changes in production and consumption patterns and their negative effects on the environment; the vicious circle between the generation of electrical energy from a matrix highly dependent on fossil sources and the frequency of extreme events such as heat waves in the most densely
} 
populated area of the country in the (AMBA) Buenos Aires. Guidelines are presented for a systemic approach considering the complexity of the environmental impact coming from energy consumption. On and off grid electricity generation with renewable energy, the development of effective methodologies to act in regulatory terms on passive techniques in buildings and affordable intervention strategies on the market regarding the sale of active thermal conditioning systems (HVAC).

Keywords: environment, climate change, energy, sustainable development, innovation, bioclimatic architecture.

\section{Calentamiento global, cambio climático y energía}

El cambio climático constituye una de las mayores amenazas ambientales de este siglo, con consecuencias económicas, sociales y ambientales de gran escala. El clima global se ha visto alterado principalmente por la quema de combustibles fósiles y los cambios en el uso de la tierra, lo que derivó en un crecimiento exponencial de la liberación de dióxido de carbono y otros gases de efecto invernadero (GEI) a la atmósfera desde el inicio de la Revolución Industrial en el siglo XVIII. El pasado más reciente es tanto más preocupante: según el Informe del Panel Intergubernamental de Expertos sobre el Cambio Climático (IPCC, por sus siglas en inglés) las emisiones de $\mathrm{CO} 2$ procedentes del uso de combustibles fósiles y los procesos industriales contribuyeron en torno al $78 \%$ del aumento total de emisiones de GEI de 1970 a 2010, con una contribución porcentual similar para el aumento experimentado durante el período de 2000 a 2010 (IPCC 2014;5). De todo el CO2 emitido, cerca de $2 / 3$ provienen de la producción de energía eléctrica, calor y transporte. Como consecuencia no deseada, se prevé la aceleración de los procesos de desertificación, una menor eficiencia del uso del agua por parte de los sistemas ecológicos y la disminución de la capacidad productiva a raíz del desgaste del suelo y sus nutrientes.

En lo que respecta al sector de edificios, el último informe elaborado por la Agencia Internacional de Energía y el Programa de las Naciones Unidas para el Medio Ambiente (IEA y UNEP por sus siglas en inglés) indica que las emisiones del sector fueron de 9.7 GtCO2 en 2018, lo que implica un aumento del 2 por ciento con respecto a 2017 y del 7 por ciento con respecto a 2010. La Alianza Global para Edificios y Construcción (GABC, 2019a) vaticina en este sentido un acelerado crecimiento: "Los edificios consumen más de un tercio de la energía final global. Se espera que la demanda de energía del sector de la construcción crezca otro 50\% para 2050".

Asimismo, el uso de energía para la refrigeración de espacios está creciendo más rápido que cualquier otro uso final de energía en edificios, llegando a ser más del triple entre 1990 y 2016 (IEA, 2018, p. 11). 


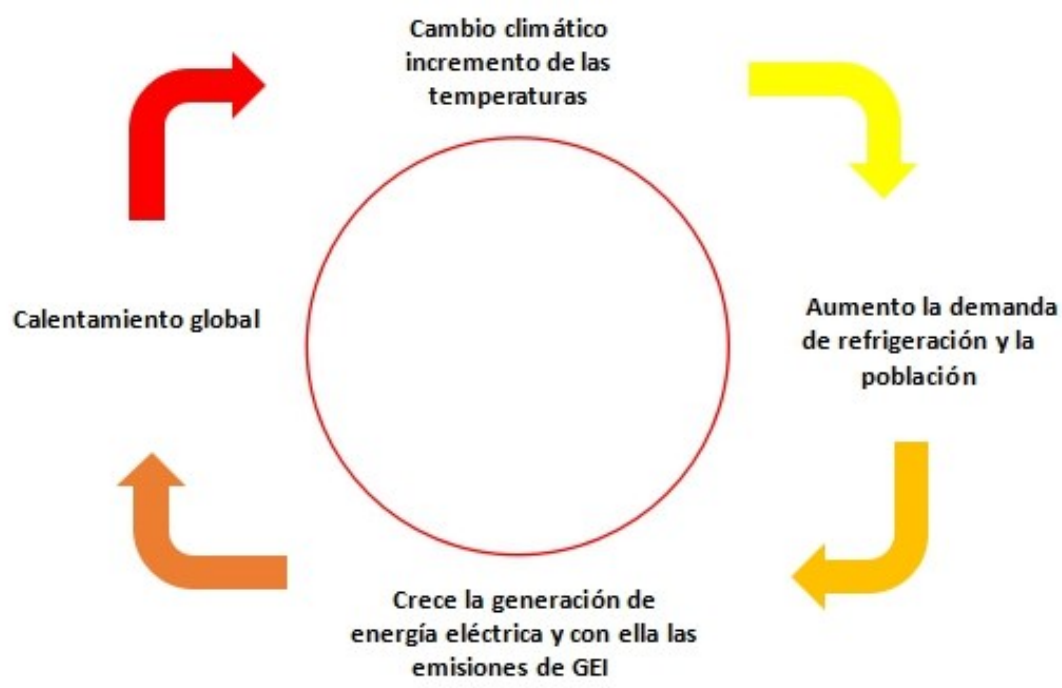

Figura 1: Circulo vicioso del uso de energías fósiles. Fuente: Elaboración propia.

Dada la actual matriz energética basada mayormente en combustibles fósiles, el círculo vicioso entre calentamiento global y generación de energía pareciera ser fatalmente perfecto (véase Figura 1).

La demanda de energía para refrigerar espacios en edificios ha experimentado un aumento notorio a nivel global: es responsable de cerca del 50 por ciento (o más) de la demanda pico de electricidad residencial, tal como lo demuestran los picos diarios en Beijing durante la ola de calor del verano de 2017, en Europa en 2003, en Australia en 2019 y análogamente en Argentina en diciembre de 2013 y 2015. En algunas regiones esta demanda creciente de refrigeración está impactando de manera negativa en la capacidad de generación y distribución de energía, especialmente durante los períodos de demanda pico y los eventos de calor extremo.

Tomando como ejemplo la ciudad de Buenos Aires las olas de calor han visto triplicada su frecuencia y duplicada su duración máxima en días en el periodo por décadas que va de 1960 a 2020 (I. Camilioni, 2012), entendiendo por ola de calor la persistencia durante varios días de temperaturas mínimas superiores a los $21^{\circ} \mathrm{C}$ y máximas por encima de los $32^{\circ} \mathrm{C}$. Las temperaturas más elevadas se registran en el área central de la ciudad debido a la densidad y compacidad de las construcciones, con valores que tienden a disminuir hacia la periferia. Este fenómeno, conocido como "isla de calor", se produce a raíz de una serie de factores:

- El almacenamiento de calor debido a la inercia térmica de los materiales que constituyen la masa edilicia.

- La producción de calor derivado de las actividades humanas en la ciudad. De acuerdo con el principio de degradación de la energía, cuando se efectúa una 
transformación de la energía siempre surge energía térmica, es decir, hay una pérdida de energía en forma de calor no utilizable que es liberada al ambiente inmediato.

- Disminución de la evaporación a causa de la reducción de áreas verdes, la impermeabilización de suelos urbanos y la escorrentía, que impiden el ciclo natural del agua y el almacenamiento del recurso en el suelo.

- Reducción de la velocidad del viento en ambientes urbanos.

- Mayor absorción de la radiación solar. Gran cantidad de superficie expuesta debido a la geometría urbana. Se produce albedo con múltiples reflexiones de la radiación solar incidente.

- Remisión de radiación térmica infrarroja por contaminación del aire urbano.

Las proyecciones no son alentadoras, más aun considerando que las condiciones climáticas arrojadas por los diversos escenarios marcan un claro aumento de las olas de calor que potenciarían esos mismos factores.

En el paper citado I.Camilioni (2012) se presentan resultados de estudios a partir del ensamble de varios modelos climáticos globales (Coupled Model Intercomparison Project, CMIP5) en el marco del Quinto Informe de Evaluación del IPCC, en el cual se consideran cuatro escenarios posibles con cuatro cortes temporales para describir las actividades antrópicas que actúan como forzantes para cambios en el clima futuro, tanto a nivel anual como estacional, para la ciudad de Buenos Aires. Los escenarios fueron nombrados de acuerdo con el forzamiento radiativo ${ }^{1}(\mathrm{w} / \mathrm{m} 2)$ que producen y las concentraciones de CO2eq atmosférico (ppm). Para todos los casos se identifica un aumento de la temperatura media anual con magnitudes que se incrementan en los escenarios más extremos y la evolución temporal del índice noches cálidas con valores que duplican los registrados en el periodo de referencia.

Simultáneamente, las emisiones de $\mathrm{CO} 2$ por enfriamiento de espacios también se están expandiendo rápidamente: entre 1990 y 2018 se triplicaron a pesar de las mejoras en el rendimiento promedio de equipos de aire acondicionado (AA) y la intensidad de carbono del sector eléctrico (IEA, 2018, p. 11).

Esa tendencia se inscribe dentro de un sector de por sí fuertemente emisor de GEI: los edificios representan el 28 por ciento de emisiones mundiales de $\mathrm{CO} 2$ relacionadas con la energía (39 por ciento si se incluyen las emisiones provenientes de la industria de la construcción) (GABC, 2019b), y las emisiones indirectas (es decir, de la generación de

\footnotetext{
${ }^{1}$ Cambio en el flujo neto de energía radiativa hacia la superficie de la Tierra medido en el tope de la tropósfera (aproximadamente 12 kilómetros sobre el nivel del mar) como resultado de cambios en la composición de la atmósfera, o cambios en el aporte externo de energía solar.
} 
energía para electricidad y calor) representan la mayor parte de las emisiones de $\mathrm{CO} 2$ relacionadas con la energía en el sector.

\section{Energía y edificios en Argentina}

El sector energético tiene una fuerte influencia en el calentamiento global, siendo la principal fuente de emisiones de GEI a nivel mundial. De acuerdo con datos de la tercera Comunicación Nacional de la República Argentina, en nuestro país el 53\% del total de las emisiones proviene de este sector (SGAyDS, 2015).

La oferta interna de energía primaria en Argentina está compuesta en un $89 \%$ por hidrocarburos ( $57 \%$ gas, $31 \%$ petróleo, $1 \%$ carbón). La generación térmica representa aproximadamente entre un $60 \%$ a un $65 \%$ del total de la electricidad generada (SE. BEN, 2018a) (SGAyDS, 2019). Los recursos energéticos están, mayoritariamente, localizados lejos de los centros de consumo, por lo que se necesita una gran infraestructura para abastecer la demanda.

\section{Ciudades, edificios y energía}

Dentro de la matriz de consumo final los edificios se llevan un $36 \%$ del total de la energía $-29 \%$ residencial, $7 \%$ otros edificios. La fuente principal de consumo de energía en edificios está relacionada con el acondicionamiento térmico interior y climatización, una proporción que en promedio asciende a un $60 \%$, teniendo en cuenta que varía notablemente de acuerdo con la región climática del país y los ciclos de frio (calefacción) y calor (refrigeración) (Secretaría de Energía, 2018b).

\section{Representatividad ambiental, densidad y alcance}

La gran diversidad de condiciones climáticas de Argentina, contando particularmente con mayor variedad respecto a otros países de la región, es resultado de 3 factores:

- Latitud: La distancia Norte-Sur del Sector Continental de Argentina excede los $3800 \mathrm{~km}$.

- Altura: Argentina también cuenta con una importante variación de alturas, desde el nivel del mar hasta 5000 metros, con ciudades como La Quiaca a 3500 m de altura sobre el nivel del mar.

- Distancia al mar: Importantes diferencias respecto de la distancia al mar, conformando zonas con influencia marítima y otras muy continentales de gran amplitud térmica, tanto diaria como anual. 
De acuerdo con datos del Tercer Informe Bienal de Actualización para la República Argentina el $91 \%$ de la población es urbana, concentrándose el $32 \%$ del total de la población en el Área Metropolitana de Buenos Aires (AMBA) (SGAyDS, 2019). La Región Sur, correspondiente a la Patagonia argentina, presenta la mayor demanda de energía para acondicionamiento térmico interior por las condiciones invernales extremas. Por la duración y severidad de la época de invierno se requiere 4,5 veces más energía para calefaccionar una vivienda construida en Ushuaia comparativamente con la misma vivienda en Buenos Aires. Sin embargo, a pesar de que la región ocupa aproximadamente un $40 \%$ del territorio nacional, la densidad poblacional es muy baja: las cinco provincias que conforman la Patagonia reúnen solo el $5 \%$ de la población del país. La mayor parte de la población se encuentra en la región central, en torno a las zonas templadas correspondientes con regiones bioambientales III y IV (IRAM 11603).

Se concluye que si bien la inversión en mejoras de envolvente térmica en las zonas bioclimáticas de clima frío y muy frío logran mayores beneficios en el ahorro de energía para calefacción, las mejoras de este tipo en zonas de clima cálido y templado alcanzan a una mayor proporción de la población, además de sus beneficios sociales relacionados, por mejorar las condiciones de confort en verano.

\section{Ciudad de Buenos Aires (AMBA), clima y confort térmico de invierno y verano}

Condiciones de verano: Las temperaturas típicas oscilan entre 16,4 y 28,5 grados, con una máxima absoluta de $41,2{ }^{\circ} \mathrm{C}$. Estas temperaturas están combinadas con humedades relativas del $70 \%$ en promedio, aumentando la sensación de disconfort y afectando el rendimiento durante exposiciones prolongadas.

Condiciones de invierno: Las temperaturas típicas oscilan entre $1,8^{\circ} \mathrm{C}$ y $16.2^{\circ} \mathrm{C}$, con 14,4 grados de amplitud y un mínimo absoluto de $-6,0{ }^{\circ} \mathrm{C}$. Estas temperaturas se encuentran por debajo de los límites mínimos de confort. Todos los meses del año presentan temperaturas medias frías durante la noche, aunque durante el día más de la mitad de los meses cuentan con temperaturas confortables. 


\begin{tabular}{|c|c|c|c|}
\hline & Latitud & Longitud & Altura \\
\hline Datos climaticos Aeroparque de Buenos Aires & $34^{\circ} 33^{\prime} 31^{\prime \prime} \mathrm{S}$ & $58^{\circ} 24^{\prime} 52^{\prime \prime} \mathrm{W}$ & $9 \mathrm{~m}$ \\
\hline Temperatura media invierno & \multicolumn{3}{|c|}{$12,91 \stackrel{\circ}{C}$} \\
\hline Temperatura media máxima invierno & \multicolumn{3}{|c|}{$17,1 \stackrel{\circ}{C}$} \\
\hline Temperatura media mínima invierno & \multicolumn{3}{|c|}{ 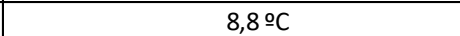 } \\
\hline Temperatura mínima de diseño, invierno & \multicolumn{3}{|c|}{$0,1 \stackrel{\circ}{\circ}$} \\
\hline Temperatura mínima absoluta, invierno & \multicolumn{3}{|c|}{$*-2,1 \stackrel{\circ}{\circ}$} \\
\hline Precipitación, media, meses de invierno, & \multicolumn{3}{|c|}{$\mathrm{mm} 278$} \\
\hline Humedad relativa, invierno & \multicolumn{3}{|c|}{$77 \%$} \\
\hline Heliofanía relativa, invierno & \multicolumn{3}{|c|}{5} \\
\hline Velocidad media del viento, & \multicolumn{3}{|c|}{$\mathrm{km} / \mathrm{hr} 9,7$} \\
\hline Grados días de calefacción, invierno, base $16^{\circ} \mathrm{C}$ & \multicolumn{3}{|c|}{538} \\
\hline Grados días de calefacción, invierno, base $18^{\circ} \mathrm{C}$ & \multicolumn{3}{|c|}{854} \\
\hline Grados días de calefacción, invierno, base $20^{\circ} \mathrm{C}$ & \multicolumn{3}{|c|}{1249} \\
\hline Grados días de calefacción, invierno, base $22^{\circ} \mathrm{C}$ & \multicolumn{3}{|c|}{1723} \\
\hline Temperatura media verano & \multicolumn{3}{|c|}{$26,68 \stackrel{\circ}{C}$} \\
\hline Temperatura media máxima verano & \multicolumn{3}{|c|}{$28,5 \circ \mathrm{C}$} \\
\hline Temperatura media mínima verano & \multicolumn{3}{|c|}{ 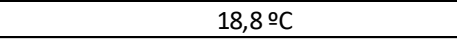 } \\
\hline Temperatura máxima de diseño, verano & \multicolumn{3}{|c|}{$36,5 \circ \mathrm{C}$} \\
\hline Temperatura máxima absoluta, verano & \multicolumn{3}{|c|}{40,5 ㅇ } \\
\hline Precipitación, media, meses de verano, mm & \multicolumn{3}{|c|}{515,8} \\
\hline Humedad relativa, verano & \multicolumn{3}{|c|}{67,6} \\
\hline Heliofanía relativa, verano & \multicolumn{3}{|c|}{8,2} \\
\hline Velocidad media del viento, $\mathrm{km} / \mathrm{hr}$ & \multicolumn{3}{|c|}{11,1} \\
\hline KT, Transparencia de la atmosfera, enero & \multicolumn{3}{|c|}{0,57} \\
\hline Radiación sobre plano horizontal, enero & \multicolumn{3}{|c|}{24,7} \\
\hline KT, Transparencia de la atmosfera, junio & \multicolumn{3}{|c|}{0,47} \\
\hline Radiación sobre plano horizontal, junio & \multicolumn{3}{|c|}{7,5} \\
\hline
\end{tabular}

Tabla 1: Elaboración propia. Datos climáticos Ciudad de Buenos Aires, Argentina. Fuente: IRAM 11603

\section{Nuevas pautas de consumo y estándares de confort térmico}

En los últimos años ha habido una gran transformación en relación con las pautas generales de consumo y estándares de confort térmico, acompañadas por notables y rápidas transformaciones tecnológicas. El avance en las tecnologías de calefacción, ventilación y aire acondicionado (HVAC, por sus siglas en inglés) y la abundante oferta en el mercado favoreció la baja de los costos poniendo a disposición masiva una serie de artefactos que, si bien son altamente eficientes y presentan un coeficiente de rendimiento (COP, por sus siglas en inglés) que arranca en 2.5 , consumen hasta 10 veces más que los sistemas pretéritos, que proponían una sensación de confort térmico mediante ventilación, es decir, a través del movimiento forzado del aire. Los ventiladores no enfrían el aire, pero producen una sensación de refrescamiento equivalente a la disminución de $2^{\circ} \mathrm{C}$ de temperatura, incrementando la evaporación de la humedad en la piel (transpiración), por lo tanto, resultan un recurso especialmente valioso, ya que logran consumir hasta un $90 \%$ menos de energía que los equipos de $\mathrm{AA}$.

El notable avance en las tecnologías de AA, que mejora sustancialmente las condiciones de confort interior en edificios, constituye un claro ejemplo de la llamada paradoja de Jevons o efecto rebote, el cual dice formalmente que mejorar la eficiencia 
disminuye el consumo instantáneo, pero incrementa el uso del modelo provocando finalmente un aumento del consumo global.

Expresión matemática de la paradoja de Jevons:

$>E$ es la eficiencia en el consumo

$>\quad C$ es el consumo instantáneo

$>\quad \mathbf{N}$ es el número de consumidores

$>\quad i$ es el estado inicial

$>\boldsymbol{f}$ es el estado final

Para una unidad, si $\mathbf{E f}>\mathbf{E i}$ entonces $\boldsymbol{C f}<\boldsymbol{C i}$

Pero puesto que $\mathbf{N f} \gg \boldsymbol{N i}$, entonces globalmente $\mathbf{C i}$. Ei . Ni $<\mathbf{C f}$. Ef . $\mathbf{N f}$

Según el último relevamiento realizado por la IEA (2019), la variación en el uso global de vectores energéticos en edificios se debe en parte al cambio en el uso final de energía, con un crecimiento de la demanda de electricidad en edificios impulsado principalmente por la refrigeración de espacios y un incremento del consumo de energía en electrodomésticos. El uso de energía para refrigeración de espacios AA aumentó en todo el mundo en más del $20 \%$ entre 2010 y 2017, mientras que la demanda de electricidad de electrodomésticos creció un $18 \%$ y la calefacción de espacios disminuyó alrededor de un $4 \%$.

\section{Buenos Aires, creciente demanda de refrigeración}

En la temporada estival, y recientemente durante períodos más extendidos, los equipos de $A A$ producen un fuerte incremento en el consumo de energía, sobre todo en las regiones centro y norte del país, lo que dispara la demanda de energía eléctrica. En la ciudad de Buenos Aires se produce una gran cantidad de contingencias, en parte porque el sistema no estaba preparado para la creciente y explosiva demanda. Esa situación deriva en falencias en la distribución como cortes, alta y baja tensión y falta de disponibilidad del servicio eléctrico, una situación que, lejos de pertenecer al pasado, puede extenderse también en la actualidad por periodos prolongados en varias zonas de la ciudad. 


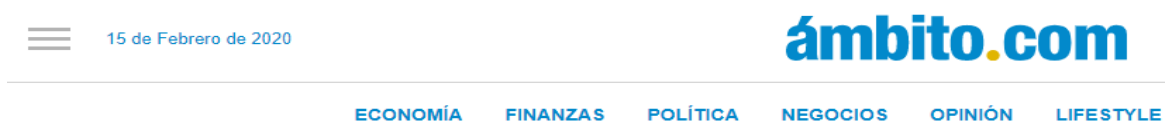

\title{
La Ciudad es un horno: la térmica llegó a los $42^{\circ}$ y rige alerta naranja por ola de calor \\ INFORMACIÓN GENERA \\ 06 Febrero 2020 \\ En Capital Federal y alrederes el calor no para y está anunciada el alerta naranja Sumado a esto, 40 mil usuarios de Edesur y Edenor se encuentran sin servicio de electricidad en la ciudad de Buenos Aires y el conurbano bonaerense
}

Últimas Noticias Podcasts Crimen de los rugbiers Dólar hoy Fotos al $100 \quad$ Registrate a nuestro Newsletter

\section{La sensación térmica llegó a $\mathbf{4 2}$ grados en la Ciudad de Buenos Aires: el ranking de temperaturas}

El Servicio Meteorológico difundió una advertencia. Recomiendan incrementar los cuidados para bebés, niños, mayores de 65 años y enfermos crónicos

\begin{abstract}
Las expectativas fueron superadas. A las 14 , la sensación térmica en la Ciudad de Buenos Aires llegó a los $\mathbf{4 2}$ grados. Con ese registro, la Capital Federal quedó tercera en el ranking de temperaturas que informa el organismo oficial detrás de Paso de los Libres, Corrientes, que llegó a los $42,3^{\circ}$ y Presidencia Roque Saenz Peña, Chaco, con 43 grados.

En la siguiente actualización, la de las 15, la térmica en la Capital Federal retrocedió a 40,5 grados y el primer lugar pasó a ser para la localidad formoseña de Las Lomitas, con 42,9 grados.
\end{abstract}

Figura 2: Medios locales con reportes del día 6 de febrero 2020.

\section{Geometría solar AMBA}

La carta estereográfica con la trayectoria solar de Buenos Aires para el período entre el 21 de diciembre y el 21 de marzo muestra que el sol sale por el sudeste y se pone por el sudoeste. Es importante considerar este factor, puesto que entonces durante los días más 
calurosos del año la radiación solar puede darse en forma directa en un espectro más amplio sobre los objetos.

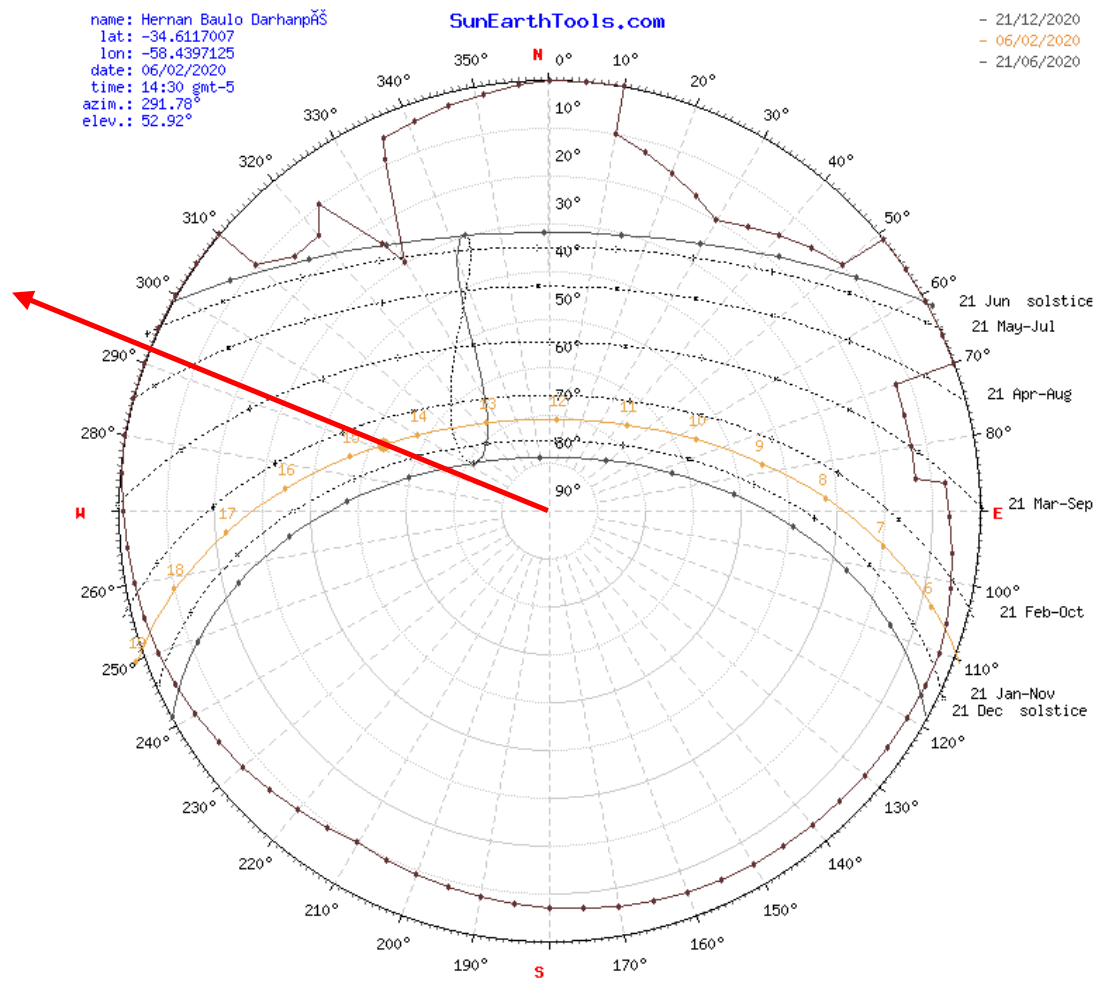

Figura 3: Elaboración propia con recurso abierto. Carta estereográfica. Acimut 291a, posición solar respecto del norte (día 6 de febrero de 2020, 14:30 h). Fuente: www.sunearthtools.com

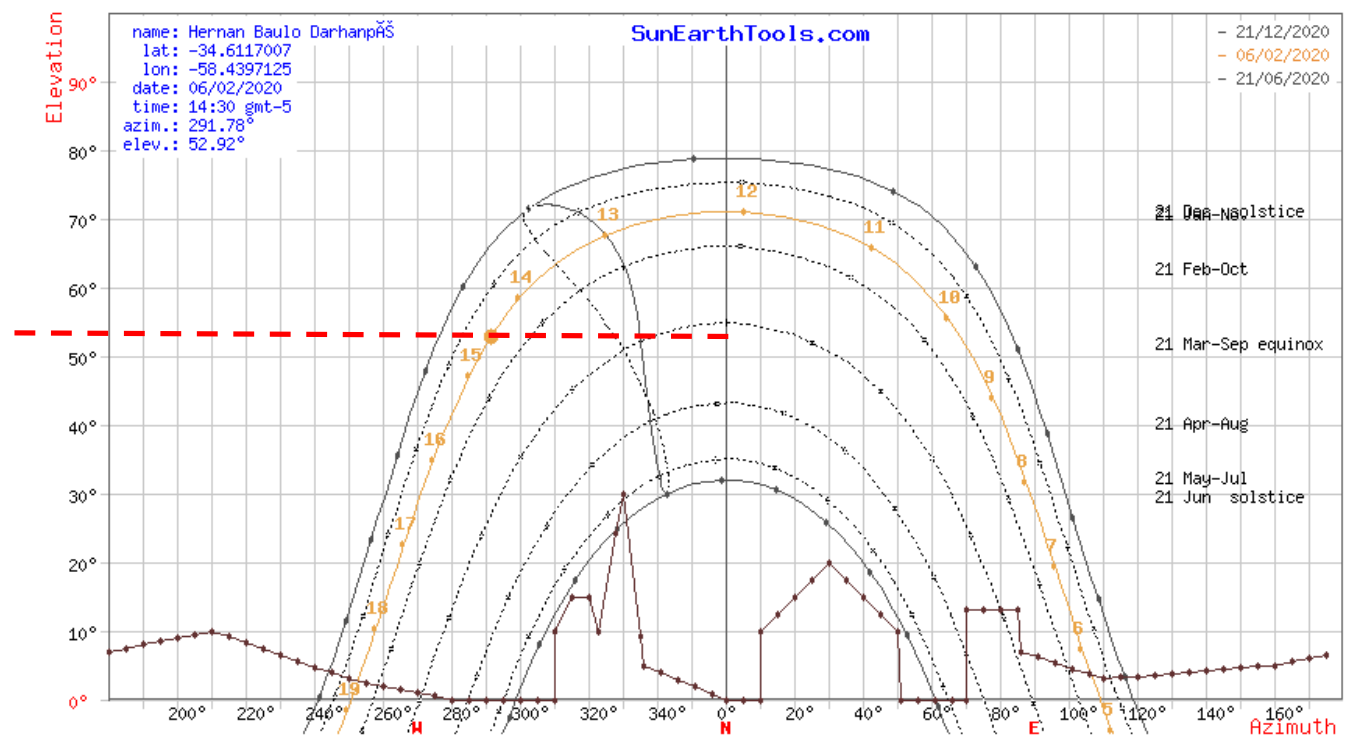

Figura 4: Elaboración propia con recurso abierto.Carta cilíndrica. Altura solar 52. 92a (día 6 de febrero de 2020. 14:30 h). Fuente: www.sunearthtools.com

La herramienta Ecotect Analysis constituye uno de entre varios soportes para proyectar arquitectura a partir de datos climáticos, dispone de archivos del clima de la 
Ciudad de Buenos Aires, enseña promedios mensuales de temperaturas en grados Celsius y la irradiancia solar en $\mathrm{W} / \mathrm{m} 2$, al igual que una franja de confort térmico para todo el año. Como se ve en la Fig. 6, en los meses de noviembre, diciembre, enero, febrero y marzo en la ciudad de Buenos Aires la temperatura de bulbo seco alcanza valores superiores a la temperatura máxima definida como límite de confort $\left(24^{\circ} \mathrm{C}\right)$, combinada con altos valores de humedad relativa con promedios superiores al $70 \%$, por lo tanto, evitar el sobrecalentamiento en el interior de los locales en estos meses se torna fundamental.

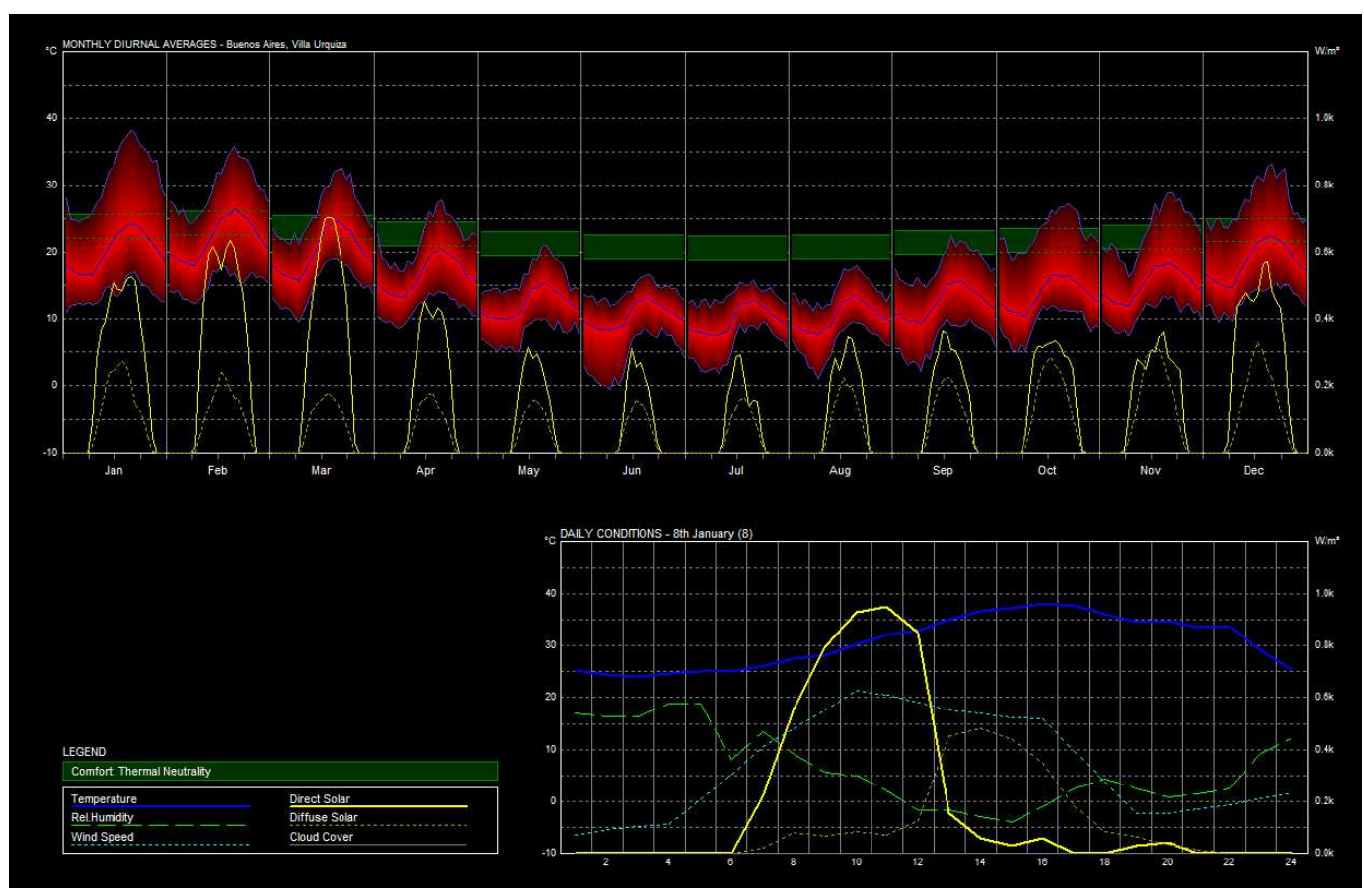

Figura 5: En la herramienta Ecotect Analysis - Weather Tool, con archivos de clima de la ciudad de Buenos Aires, la gráfica muestra promedios mensuales de temperatura en ${ }^{\circ} \mathrm{C}$ e incidencia de irradiancia solar $\mathrm{W} / \mathrm{m} 2$ en superficies. Asimismo se define una franja de confort térmico para todo el año. Se visualizan mayormente entre noviembre y marzo temperaturas por encima de la zona de confort con altos valores de radiación directa

La intensidad de la radiación solar se incrementa desde la salida del sol hasta el mediodía cuando éste se posiciona en el norte y a mayor altura, aumentando la temperatura del aire. Sin embargo, la temperatura máxima se registra más tarde debido a la capacidad térmica de la tierra, que sigue "absorbiendo" calor durante las primeras horas de la tarde. La carta muestra que poco después del mediodía, a las 14:30 hs, el sol se posiciona sobre el cuadrante oeste, con ángulos solares cada vez más bajos. Esta situación requiere un tratamiento especial en las cubiertas y en las caras de edificios expuestas a esa orientación (O - SO), dado que el sobrecalentamiento como consecuencia de esa radiación aumenta notablemente el uso de energía para refrigeración. Tal es así que en ese horario se registra el pico de demanda eléctrica en verano. Por otro lado, a 
partir de ese honorario comienza la franja horaria coincidente con el retorno de la jornada laboral, lo cual suele generar una demanda masiva de forma simultánea.

$\mathrm{Si}$ bien existen leyes y reglamentaciones que se proponen "establecer las condiciones de acondicionamiento térmico exigibles en la construcción de edificios" con el fin de "racionalizar" el uso de la energía para disminuir "el impacto ambiental" y mejorar "la calidad de vida de la población" (ley Provincia de Bs.As. 13.059/2010 o ley CABA 4458/2012), la informalidad en el sector de la construcción, los mayores costos y la dificultad en el seguimiento para que se apliquen las normas vigentes hacen que establecer nuevos estándares resulte complejo, reclamando procesos de innovación en materia de política pública para el desarrollo sostenible.

Esta falta de regularización se inscribe en un contexto global también complejo: la superficie construida de edificios en todo el mundo ha aumentado en un $65 \%$ entre 2000 y 2018. Sin embargo, el uso promedio de energía por $\mathrm{m} 2$ ha disminuido solo en un $25 \%$, lo que significa que el progreso en materia de eficiencia energética no compensó el crecimiento de la población y de la superficie construida. De acuerdo con datos de la GABC (2019), se espera que la superficie global construida se duplique para 2060, impulsando aún más la demanda de energía y las emisiones de GEI relacionadas con la construcción. El mismo informe revela que el desarrollo de mejoras en el rendimiento de las envolventes térmicas de los edificios es fundamental para lograr el Escenario de Desarrollo Sostenible (SDS por sus siglas en inglés), pero la mayoría de los países aún no las han convertido en una prioridad política explícita.

\section{Aspectos clave para la intervención.}

Es indudable que las mejoras tecnológicas han evolucionado exponencialmente en los últimos 50 años, con notables progresos que incluyen aspectos importantes de eficiencia energética. Sin embargo, las emisiones continúan en aumento al ritmo del crecimiento de la población y del PBI a nivel mundial. Cabe entonces la pregunta: ¿Puede la tecnología constituirse en la herramienta para reducir emisiones de GEls? Quizás el abordaje de la problemática desde una metodología multicriterio resulte más costo efectiva en términos ambientales frente al apremio que reclama acciones ante los escenarios tendenciales.

\section{Descarbonización del vector energético de uso en AA}

Un paso fundamental hacia una mejora sustancial es la descarbonización de la matriz eléctrica, que podría efectuarse a través de una contribución más ambiciosa de las fuentes de energía renovables (ER) en la matriz de generación. Si bien existen tecnologías 
como los chillers de absorción en base a gas natural, el vector de uso predominante en los sistemas HVAC es la electricidad.

Es de amplio conocimiento que Argentina tiene notables ventajas en cuanto a recursos para favorecer la generación de energía eléctrica con fuentes renovables, tanto debido al fuerte potencial solar en el noroeste como al claro recurso eólico en la zona terrestre y offshore de la Patagonia, recursos biomasicos, gases de vertedero, gases de plantas de depuración y biogás para generación térmica y potencial para pequeños aprovechamientos hidroeléctricos. El "Régimen de Fomento Nacional para el Uso de Fuentes Renovables de Energía Destinada a la Producción de Energía Eléctrica" (Ley 26.190) y el "Régimen de fomento a la generación distribuida de energía renovable integrada a la red eléctrica pública" (ley 27.424) establecen un marco de promoción y regulatorio nacional que actúa en favor de esas acciones.

El principal aspecto de la ley 26.190, es que introdujo por primera vez como política de acceso un objetivo: la contribución de las ER al consumo de energía eléctrica nacional debería ser del $8 \%$ en 2016. Lejos de cumplirse el objetivo de la ley promulgada en 2006 y modificaciones ley 27.191/15, la baja penetración actual de las ER se debe según numerosos autores a dificultades relacionadas con las condiciones de entorno como: la calidad institucional y el cumplimiento de las normas regulatorias, la capacidad de adaptación del país a nuevas tecnologías, el acceso al financiamiento, y el conocimiento tecnológico y capital humano, entre otras características.

\section{Promoción de sistemas pasivos}

Otro gran aporte reside en la configuración de estrategias pasivas en la construcción, es decir, la incorporación durante el diseño de recursos calibrados con el clima que actúen en contra de la demanda de sistemas activos para el acondicionamiento térmico interior. Marco de promoción con estudios de asoleamiento para el uso adecuado de:

- Protecciones solares: Considerar sistemas de protección solar como parasoles horizontales y verticales, mallas y cortinas de enrollar de color claro.

- Fachadas ventiladas: Impulsar un retrofit que contemple fachadas ventiladas en orientaciones oeste - noroeste - suroeste evitará ganancias térmicas interiores no deseadas. Del mismo modo, superficies blancas expuestas pueden reflejar hasta el $80 \%$ de la energía del sol en comparación con muros y cubiertas negras que reflejan solo del $5 \%$ al $10 \%$.

- Regulación restrictiva para proyectos con orientación oeste, como ser la exigencia de reducir las superficies de vidrio y fijar un Window wall ratio (WWR, por sus siglas en inglés) específico para dicha orientación. Asimismo, podrían establecerse normativas que fijen parámetros de superficies y proporciones coherentes expuestas a cada orientación, de 
modo de bajar las cargas reguladas para acondicionamiento térmico, reducir las ganancias térmicas no deseadas y, en consecuencia, disminuir el uso de sistemas para enfriamiento. - Desarrollo de especificaciones técnicas para sistemas pasivos de apoyo a equipos de AA en proyectos de gran escala que favorezcan una menor demanda de energía para enfriar el fluido o aprovechamientos en procesos de co-generación. Un ejemplo serían los sistemas Aire/Tierra como apoyo geotérmico a bombas de calor. El terreno que envuelve los conductos enfría en verano el aire ya que se halla a una temperatura menor antes del ingreso a la Unidad de Tratamiento de Aire.

\section{Incremento de soluciones basadas en la naturaleza}

- Layers vivos: Capas verdes y cubiertas verdes para contribuir con el ambiente urbano mediante el sostenimiento ecológico en grandes ciudades. Las capas y cubiertas favorecen la aislación térmica del edificio en cuestión, reduciendo la demanda interior de energía y contribuyen con:

- la reducción de los niveles de ruido

- la purificación del aire

- la absorción del agua de lluvia

- la reducción del efecto "isla de calor" y mejoras en la calidad del aire

Es decir, los beneficios de estas incorporaciones son tanto para el edificio en sí mismo como para el ambiente urbano inmediato.

\section{Huella hídrica}

Otro aporte no menor consistiría en abordar la acuciante problemática del agua Favorecer el desarrollo de sistemas de acumulación común en edificios multifamiliares para utilizar el condensado de bombas de calor junto con la recolección de agua de lluvia en una reserva para uso en inodoros y limpieza de veredas colaboraría con la reducción del volumen de aguas residuales y de energía destinada a bombeo e impulsión.

\section{Intervención en el mercado local de sistemas activos de acondicionamiento térmico}

Según estudios realizados por la IEA (2018), los AA de alto rendimiento que ya están disponibles en el mercado podrían reducir la demanda de refrigeración entre un $40 \%$ y un $50 \%$, disminuyendo significativamente los impactos en el sector eléctrico. El ratio de eficiencia energética estacional (SEER por sus siglas en inglés) promedio de las unidades de $A A$ vendidas en los mercados de más rápido crecimiento, como China e India, generalmente es inferior a 3.5. Sin embargo, los productos disponibles en esos mismos 
mercados, a menudo a precios comparables, pueden tener SEERs que son $50 \%$ a $70 \%$ mejores.

Una posible estrategia a nivel local, para evitar la futura ampliación de la potencia instalada para generación eléctrica ante la demanda en rápido crecimiento, podría basarse en facilitar desde el sector público la adquisición de tecnologías más eficientes ofreciendo por ejemplo opciones de financiamiento sobre la demanda como ser un plan de reconversión y reposición de unidades existentes por tecnologías más actuales como los inverter o SEERs superiores. Esto aportaría un beneficio extendido, dado que la tecnología está siendo usada de manera creciente tanto para ciclos de refrigeración en verano como de calefacción en invierno, mejorando asimismo tanto las condiciones de habitabilidad como la economía familiar para los casos residenciales. Un plan de dichas características debería ser abordado desde ya desde una perspectiva de Ciclo de Vida $(\mathrm{ACV})$, planificando muy bien el tratamiento de descarte y deshechos en el marco de la enmienda de Kigali y el Protocolo de Montreal.

\section{Conclusiones}

El presente trabajo se propone hacer aportes dando visibilidad a diversos ejes para el diseño de instrumentos de política pública en el marco de un plan de mitigación y acciones locales de adaptación al cambio climático en lo relativo al uso de energía para enfriamiento de espacios en edificios.

Como marco se presenta la relación cíclica entre las emisiones de gases de efecto invernadero (GEls) provenientes del uso de combustibles fósiles para la generación de energía, el incremento de la temperatura a nivel global y en particular el aumento en la intensidad y frecuencia de eventos extremos de calor en el AMBA. Se exponen las características propias de la morfología urbana de la ciudad de Buenos Aires, que contribuyen con el fenómeno e impulsan el crecimiento de la demanda de energía para refrigeración de espacios en edificios, lo cual sigue una tendencia mundial.

No obstante los beneficios que aportan las nuevas tecnologías y su creciente desarrollo, las emisiones de GEls continúan creciendo, lo cual demuestra que el sector no contrarresta una tendencia que se muestra en aumento. El escenario reclama múltiples acciones desde un abordaje multicritério.

Se proponen entonces como ejes fundamentales la necesidad de otorgar visibilidad a la problemática e instalar pautas de uso racional y eficiente de recursos y energía; la reducción del consumo mediante estrategias pasivas y sistemas activos eficientes que generen una disminución de la demanda hasta lograrse una transición más equilibrada 
hacia la generación de energía con recursos renovables; y modelos descentralizados que bajen la intensidad de carbono de la industria energética.

Asimismo, se plantea la incorporación de procesos de innovación a partir de métricas e indicadores de sustentabilidad consistentes, útiles para la difusión de la información acerca del consumo en edificios; la tracción en el mercado local para impulsar sistemas pasivos con uso de baja tecnología y soluciones basadas en la naturaleza. En este sentido, se señala que resulta decisivo alimentar procesos de $I+D$ para lograr una transferencia mas ágil al sector socio-productivo; generar incentivos y movilizar fondos para del desarrollo endógeno; dotar de profesionalización y formalidad al espacio que brinda productos en las tres esferas (diseño, desarrollo de materiales y construcción y uso u operación) y profesionalizar un área que brinde modelos cuantitativos que permitan desarrollos clara y comprobablemente acordes con un modelo de desarrollo sostenible local en el amplio mercado de la construcción y operación de edificios.

\section{Referencias bibliográficas}

Hannart, C. Vera, F. E. L. Otto y otros. (2015) Causal influence of anthropogenic forcings on the Argentinian heat wave of december 2013. paper from: Explaining Extreme Events of 2014 from a Climate Perspective. Bulletin of the American Meteorological Society.

I. Camilloni (2012). Cambio climático en la ciudad de Buenos Aires: cambios observados y escenarios futuros. Recuperado (9/10/20)

https://www.buenosaires.gob.ar/sites/gcaba/files/informe camilloni1.pdf

IPCC (2015). Cambio climático 2014. Mitigación del cambio climático. Resumen para responsables de políticas. Grupo Intergubernamental de Expertos sobre el Cambio $\begin{array}{lllll}\text { Climático. } & \text { [Archivo PDF] (7- } & 9) & \text { Recuperado } & \text { (02/09/20) }\end{array}$ https://archive.ipcc.ch/home_languages main_spanish.shtml

IPCC, (2014). Cambio climático 2014: Informe de síntesis. Contribución de los Grupos de trabajo I, II y III al Quinto Informe de Evaluación del Grupo Intergubernamental de Expertos sobre el Cambio Climático [Equipo principal de redacción, R.K. Pachauri y L.A. Meyer (eds.)]. IPCC, Ginebra. Recuperado https://www.ipcc.ch/site/assets/uploads/2018/02/SYR AR5 FINAL full es.pdf

$(15 / 10 / 20)$

IEA (2018) The Future of Cooling. Opportunities for energyefficient air conditioning [Archivo PDF]. Recuperado (10/10/20): https://webstore.iea.org/download/direct/1036

IEA (2019) World Energy Statistics and Balances and Energy Technology Perspectives. Recuperado (9/09/2019): https://www.iea.org/subscribe-to-data-services/world-energybalances-and-statistics

GABC (2019). Global Status Report for Buildings and Construction (International Energy Agency - United Nations Environment Programme) [Archivo PDF] (9 - 16). Recuperado (02/09/20): https://globalabc.org/ 
Secretaría de Ambiente y Desarrollo Sustentable (SGAyDS). (2015). 3ra. Comunicación Nacional de la República Argentina a la Convención Marco de las Naciones Unidas sobre el Cambio Climático. [Archivo PDF]. Buenos Aires: SAyDS. Recuperado de https://unfccc.int/resource/docs/natc/argnc3s.pdf

Secretaría de Ambiente y Desarrollo Sustentable (SGAyDS). (2019). Tercer Informe Bienal de Actualización de Argentina a la Convención Marco de las Naciones Unidas para el Cambio Climático (CMNUCC). [Archivo PDF] (20 - 23) Recuperado (28/08/20) https://www.argentina.gob.ar/sites/default/files/3er iba.pdf

Secretaria de Energía (SE) (2018). Balance Energético Nacional (BEN) Revisión 2. Recuperado https://www.argentina.gob.ar/economia/energia/hidrocarburos/balancesenergeticos

Norma IRAM 11603. (2012). Acondicionamiento térmico de edificios. Clasificación Bioambiental de la República Argentina. Buenos Aires: IRAM.

Norma IRAM 11605. (1996). Acondicionamiento térmico de edificios. Condiciones de habitabilidad en edificios. Valores máximos de la transmitancia térmica en cerramientos opacos. Buenos Aires: IRAM.

S, Gil y otros (2017). Eficiencia en el calentamiento de agua caliente sanitaria en Argentina. Uso de la Energía en el sector residencial.

Wikipedia < William Stanley Jevons > https://es.wikipedia.org/wiki/Paradoja de Jevons

Sun Earth tools. (2019) Elaboración propia en base a https://www.sunearthtools.com/

Ley 13.059/2003. Acondicionamiento Térmico de Edificios. Recuperado https://normas.gba.gob.ar/ar-b/ley/2003/13059/3792

Ley 4458/2012 Normas de acondicionamiento térmico en la construcción de edificios. Recuperado https://boletinoficial.buenosaires.gob.ar/normativaba/norma/218635

Ley 27191/2015. Modificaciones a la Ley 26.190/2006, "Régimen de Fomento Nacional para el Uso de Fuentes Renovables de Energía Destinada a la Producción de Energía Eléctrica". http://servicios.infoleg.gob.ar/infoleglnternet/anexos/250000254999/253626/norma.htm

Ley 27424/2017. Régimen de fomento a la generación distribuida de energía renovable integrada a la red eléctrica pública. Recuperado http://servicios.infoleg.gob.ar/infolegInternet/verNorma.do?id=305179 